International Journal of Applied Mathematics

Volume 34 No. $6 \quad 2021,1031-1047$

ISSN: 1311-1728 (printed version); ISSN: 1314-8060 (on-line version) doi: http://dx.doi.org/10.12732/ijam.v34i6.1

\title{
A NOVEL KIND OF THE MULTI-INDEX WHITTAKER FUNCTION AND ITS CERTAIN PROPERTIES
}

\author{
Musharraf Ali ${ }^{1}$, Mohd Ghayasuddin ${ }^{2}$, Waseem A. Khan ${ }^{3}$, \\ Kottakkaran S. Nisar ${ }^{\S}$ \\ ${ }^{1}$ Department of Mathematics \\ G.F. College \\ Shahjahanpur - 242001, INDIA \\ 2 Department of Mathematics \\ Integral University \\ Centre Shahjahanpur - 242001, INDIA \\ ${ }^{3}$ Department of Mathematics and Natural Sciences \\ Prince Mohammad Bin Fahd University, P.O. Box: 1664 \\ Al Khobar - 31952, SAUDI ARABIA \\ ${ }^{4}$ Department of Mathematics, College of Arts and Sciences \\ Prince Sattam bin Abdulaziz University \\ Wadi Aldawaser, SAUDI ARABIA
}

\begin{abstract}
In this paper, we propose a novel expansion of the Whittaker function $M_{\eta, \zeta}(w)$ by utilizing the known expanded confluent hypergeometric function of the first kind $\Phi_{p}^{\left(\lambda_{1}, \cdots, \lambda_{s}, \delta_{1}, \cdots, \delta_{s}\right)}\left(\kappa_{2} ; \kappa_{3} ; w\right)$. Moreover, some integral representations, transformation formulae, integral transforms and a differential formula also established.
\end{abstract}

AMS Subject Classification: $33 \mathrm{C} 15,33 \mathrm{C} 05,33 \mathrm{~B} 15$

Key Words: Whittaker function; expanded Whittaker function; Gauss hypergeometric function; confluent hypergeometric function; expanded confluent hypergeometric function; Beta function; expanded Beta function

Received: June 15, 2020

(C) 2021 Academic Publications

${ }^{\S}$ Correspondence author 


\section{Introduction and Preliminaries}

Special functions are an ancient part of Mathematics, as solutions of a wide class of Mathematically and Physically relevant models. These functions play an important role in many fields of science and engineering. Many researchers put efforts and achievements to study and develop their theory and applications. Different expansions of the traditional special functions for example, Beta function, hypergeometric function, Whittaker function, have been proposed and investigated by many researchers, to be specific, Chaudhry et al. $[4,5]$, Lee et al. [14], Özergin et al. [19], Choi et al. [2], Parmar [23], Dar et al. [6], Shadab et al. [28], Mubeen et al. [16] and Nagar et al. [18]. A list of Whittaker functions have been explored (see, for example, [1], [3], [6], [7], [18], [26], [27] and the references therein). In this sequel, we expect to present a new generalization of the Whittaker function of the first kind as far as summed up confluent hypergeometric function introduced by Ghayasuddin et al. [10].

First, we need to survey here the definitions of some known special functions and their extensions.

Let us start with some historical background of the Euler classical Beta function. The function $B\left(\xi_{1}, \xi_{2}\right)$ given by

$$
\begin{gathered}
B\left(\xi_{1}, \xi_{2}\right)=\int_{0}^{1} u^{\xi_{1}-1}(1-u)^{\xi_{2}-1} d u \\
\left(\Re\left(\xi_{1}\right)>0, \Re\left(\xi_{2}\right)>0\right),
\end{gathered}
$$

was defined and first studied by Leonhard Euler in the 18th century and was given its name by Jacques Binet (see [25], [29], see also [15]).

Its relation with well-known classical Gamma function (see [25], see also [29])

$$
\Gamma\left(\xi_{1}\right)=\int_{0}^{\infty} u^{\xi_{1}-1} e^{-u} d u \quad\left(\Re\left(\xi_{1}\right) \geq 0\right)
$$

is given by

$$
B\left(\xi_{1}, \xi_{2}\right)=\frac{\Gamma\left(\xi_{1}\right) \Gamma\left(\xi_{2}\right)}{\Gamma\left(\xi_{1}+\xi_{2}\right)} \quad\left(\xi_{1}, \xi_{2} \in C \backslash Z_{0}^{-}\right),
$$

where $C$ and $Z_{0}^{-}$indicate the well defined collection of complex numbers and non-positive integers, respectively.

The classical Gauss hypergeometric function ${ }_{2} F_{1}$ and the confluent hypergeometric function of first kind ${ }_{1} \Phi_{1}$ are defined by (see [25], see also [29])

$$
{ }_{2} F_{1}\left(\kappa_{1}, \kappa_{2} ; \kappa_{3} ; w\right)=\sum_{\ell=0}^{\infty} \frac{\left(\kappa_{1}\right)_{\ell}\left(\kappa_{2}\right)_{\ell}}{\left(\kappa_{3}\right)_{\ell}} \frac{w^{\ell}}{\ell !}
$$




$$
\left(\kappa_{1}, \kappa_{2} \in C \text { and } \kappa_{3} \in C \backslash Z_{0}^{-} ; w \in C\right)
$$

and respectively, by

$$
\begin{gathered}
{ }_{1} F_{1}\left(\kappa_{2} ; \kappa_{3} ; w\right)=\sum_{\ell=0}^{\infty} \frac{\left(\kappa_{2}\right)_{\ell}}{\left(\kappa_{3}\right)_{\ell}} \frac{w^{\ell}}{\ell !} \\
\left(\kappa_{2} \in C \text { and } \kappa_{3} \in C \backslash Z_{0}^{-} ; w \in C\right),
\end{gathered}
$$

where $\left(\kappa_{1}\right)_{\ell}=\frac{\Gamma\left(\kappa_{1}+\ell\right)}{\Gamma\left(\kappa_{1}\right)}$ denotes the Pochammer symbol, or rising factorial (see, e.g. [30]).

Leonhard Euler proposed the following integral representations of the Gauss hypergeometric function ${ }_{2} F_{1}$ and of the confluent hypergeometric function of first kind ${ }_{1} \Phi_{1}$ (see [25] and [30]):

$$
\begin{gathered}
F\left(\kappa_{1}, \kappa_{2} ; \kappa_{3} ; w\right)=\frac{1}{B\left(\kappa_{2}, \kappa_{3}-\kappa_{2}\right)} \int_{0}^{1} u^{\kappa_{2}-1}(1-u)^{\kappa_{3}-\kappa_{2}-1}(1-u w)^{-\kappa_{1}} d u \\
\left(\Re\left(\kappa_{3}\right)>\Re\left(\kappa_{2}\right)>0 \quad ;|\arg (1-w)| \leq \pi-\epsilon \quad(0<\epsilon<\pi)\right),
\end{gathered}
$$

or equivalently

$$
\begin{gathered}
F\left(\kappa_{1}, \kappa_{2} ; \kappa_{3} ; w\right)=\frac{1}{B\left(\kappa_{1}, \kappa_{3}-\kappa_{1}\right)} \int_{0}^{1} u^{\kappa_{1}-1}(1-u)^{\kappa_{3}-\kappa_{1}-1}(1-u w)^{-\kappa_{2}} d u \\
\left(\Re\left(\kappa_{3}\right)>\Re\left(\kappa_{1}\right)>0 ;|\arg (1-w)| \leq \pi-\epsilon \quad(0<\epsilon<\pi)\right)
\end{gathered}
$$

and

$$
\begin{gathered}
\Phi\left(\kappa_{2} ; \kappa_{3} ; w\right)=\frac{1}{B\left(\kappa_{2}, \kappa_{3}-\kappa_{2}\right)} \int_{0}^{1} u^{\kappa_{2}-1}(1-u)^{\kappa_{3}-\kappa_{2}-1} \exp (u w) d u \\
\left(\Re\left(\kappa_{2}\right)>\Re\left(\kappa_{1}\right)>0\right) .
\end{gathered}
$$

By utilizing the arrangements of $(1-u w)^{-\kappa_{1}}$ and $\exp (u w)$ in (5) and (6) respectively, we acquire

$$
\begin{gathered}
F\left(\kappa_{1}, \kappa_{2} ; \kappa_{3} ; w\right)=\sum_{\ell=0}^{\infty}\left(\kappa_{1}\right)_{\ell} \frac{B\left(\kappa_{2}+\ell, \kappa_{3}-\kappa_{2}\right)}{B\left(\kappa_{2}, \kappa_{3}-\kappa_{2}\right)} \frac{w^{\ell}}{\ell !} \\
\left(\Re\left(\kappa_{3}\right)>\Re\left(\kappa_{2}\right)>0,|w|<1\right)
\end{gathered}
$$

and

$$
\Phi\left(\kappa_{2} ; \kappa_{3} ; w\right)=\sum_{\ell=0}^{\infty} \frac{B\left(\kappa_{2}+\ell, \kappa_{3}-\kappa_{2}\right)}{B\left(\kappa_{2}, \kappa_{3}-\kappa_{2}\right)} \frac{w^{\ell}}{\ell !}
$$




$$
\left(\Re\left(\kappa_{3}\right)>\Re\left(\kappa_{2}\right)>0,|w|<1\right) .
$$

In 1997, Chaudhry et al. [4] proposed the p-extension of the Eulerian integral of first kind by introducing the exponential factor $\exp \left[\frac{-p}{u(1-u)}\right]$ in the integrand of (1):

$$
\begin{aligned}
B_{p}\left(\xi_{1}, \xi_{2}\right)= & \int_{0}^{1} u^{\xi_{1}-1}(1-u)^{\xi_{2}-1} \exp \left[\frac{-p}{u(1-u)}\right] d u \\
& \left(\Re\left(\xi_{1}\right)>0, \Re\left(\xi_{2}\right)>0, \Re(p)>0\right) .
\end{aligned}
$$

For $p=0,(9)$ reduces to the classical beta function $B\left(\xi_{1}, \xi_{2}\right)$, i.e.

$$
B_{0}\left(\xi_{1}, \xi_{2}\right)=B\left(\xi_{1}, \xi_{2}\right) .
$$

Afterwards, Chaudhry et al. [5] utilized the expanded beta function $B_{p}\left(\xi_{1}, \xi_{2}\right)$ to extend the Gauss and the confluent hypergeometric functions as follows:

$$
\begin{gathered}
F_{p}\left(\kappa_{1}, \kappa_{2} ; \kappa_{3} ; w\right)=\sum_{\ell=0}^{\infty}\left(\kappa_{1}\right)_{\ell} \frac{B_{p}\left(\kappa_{2}+\ell, \kappa_{3}-\kappa_{2}\right)}{B\left(\kappa_{2}, \kappa_{3}-\kappa_{2}\right)} \frac{w^{\ell}}{\ell !} \\
\left(\Re\left(\kappa_{3}\right)>\Re\left(\kappa_{2}\right)>0, p \geq 0,|w|<1\right)
\end{gathered}
$$

and

$$
\begin{gathered}
\Phi_{p}\left(\kappa_{2} ; \kappa_{3} ; w\right)=\sum_{\ell=0}^{\infty} \frac{B_{p}\left(\kappa_{2}+\ell, \kappa_{3}-\kappa_{2}\right)}{B\left(\kappa_{2}, \kappa_{3}-\kappa_{2}\right)} \frac{w^{\ell}}{\ell !} \\
\left(\Re\left(\kappa_{3}\right)>\Re\left(\kappa_{2}\right)>0, p \geq 0\right) .
\end{gathered}
$$

In the same paper, the authors additionally defined the subsequent Euler's type integral representations of $F_{p}\left(\kappa_{1}, \kappa_{2} ; \kappa_{3} ; w\right)$ and $\Phi_{p}\left(\kappa_{2} ; \kappa_{3} ; w\right)$, respectively:

$$
\begin{gathered}
F_{p}\left(\kappa_{1}, \kappa_{2} ; \kappa_{3} ; w\right)=\frac{1}{B\left(\kappa_{2}, \kappa_{3}-\kappa_{2}\right)} \\
\times \int_{0}^{1} u^{\kappa_{2}-1}(1-u)^{\kappa_{3}-\kappa_{2}-1}(1-u w)^{-\kappa_{1}} \exp \left[\frac{-p}{u(1-u)}\right] d u \\
\left(\Re\left(\kappa_{3}\right)>\Re\left(\kappa_{2}\right)>0, p \geq 0,|\arg (1-w)|<\pi\right)
\end{gathered}
$$

and

$$
\begin{aligned}
\Phi_{p}\left(\kappa_{2} ; \kappa_{3} ; w\right)= & \frac{1}{B\left(\kappa_{2}, \kappa_{3}-\kappa_{2}\right)} \int_{0}^{1} u^{\kappa_{2}-1}(1-u)^{\kappa_{3}-\kappa_{2}-1} \\
& \times \exp \left[u w-\frac{p}{u(1-u)}\right] d u
\end{aligned}
$$




$$
\left(\Re\left(\kappa_{3}\right)>\Re\left(\kappa_{2}\right)>0, p \geq 0\right) .
$$

On setting $p=0$ in (10)-(13), we get the series and integral representations of the classical Gauss and confluent hypergeometric functions.

In 2018, Shadab et al. [28] introduced a new generalization of Beta function as follows:

$$
\begin{gathered}
B_{\alpha}^{p}\left(\xi_{1}, \xi_{2}\right)=\int_{0}^{1} u^{\xi_{1}-1}(1-u)^{\xi_{2}-1} E_{\alpha}\left[-\frac{p}{u(1-u)}\right] d u \\
\left(\alpha \in R_{0}^{+}, \Re(p) \geq 0\right),
\end{gathered}
$$

where $E_{\alpha}($.$) is the classical Mittag-Leffler function of one parameter which is a$ natural extension of the exponential function defined by Gosta Mittag-Leffler as follows (see [17]):

$$
\begin{gathered}
E_{\alpha}(u)=\sum_{\ell=0}^{\infty} \frac{u^{\ell}}{\Gamma(1+\alpha \ell)} \\
\left(u \in C \text { and } \alpha \in R_{0}^{+}\right) .
\end{gathered}
$$

If we put $\alpha=1 \mathrm{in}$ (14) then we get the expanded beta function defined by (9), which further for $p=0$ gives the classical Beta function defined by (1).

Shadab et al. [28] considered the following expanded hypergeometric function and expanded confluent hypergeometric function, by

$$
\begin{gathered}
F_{p, \alpha}\left(\kappa_{1}, \kappa_{2} ; \kappa_{3} ; w\right)=\sum_{\ell=0}^{\infty}\left(\kappa_{1}\right)_{\ell} \frac{B_{\alpha}^{p}\left(\kappa_{2}+\ell, \kappa_{3}-\kappa_{2}\right)}{B\left(\kappa_{2}, \kappa_{3}-\kappa_{2}\right)} \frac{w^{\ell}}{\ell !} \\
\left(\Re\left(\kappa_{3}\right)>\Re\left(\kappa_{2}\right)>0, \alpha \in R^{+}, p \in R_{0}^{+},|w|<1\right)
\end{gathered}
$$

and

$$
\begin{gathered}
\Phi_{p, \alpha}\left(\kappa_{2} ; \kappa_{3} ; w\right)=\sum_{\ell=0}^{\infty} \frac{B_{\alpha}^{p}\left(\kappa_{2}+\ell, \kappa_{3}-\kappa_{2}\right)}{B\left(\kappa_{2}, \kappa_{3}-\kappa_{2}\right)} \frac{w^{\ell}}{\ell !} \\
\left(\Re\left(\kappa_{3}\right)>\Re\left(\kappa_{2}\right)>0, p \in R_{0}^{+}, \alpha \in R^{+}\right) .
\end{gathered}
$$

The authors in [28] obtained the integral representations of the expanded Gauss and confluent hypergeometric functions as follows:

$$
\begin{gathered}
F_{p, \alpha}\left(\kappa_{1}, \kappa_{2} ; \kappa_{3} ; w\right)=\frac{1}{B\left(\kappa_{2}, \kappa_{3}-\kappa_{2}\right)} \\
\times \int_{0}^{1} u^{\kappa_{2}-1}(1-u)^{\kappa_{3}-\kappa_{2}-1}(1-u w)^{-\kappa_{1}} E_{\alpha}\left[\frac{-p}{u(1-u)}\right] d u
\end{gathered}
$$




$$
\left(\Re\left(\kappa_{3}\right)>\Re\left(\kappa_{2}\right)>0, \alpha \in R^{+}, p \in R_{0}^{+},|\arg (1-w)|<\pi\right)
$$

and

$$
\begin{aligned}
& \Phi_{p, \alpha}\left(\kappa_{2} ; \kappa_{3} ; w\right)=\frac{1}{B\left(\kappa_{2}, \kappa_{3}-\kappa_{2}\right)} \int_{0}^{1} u^{\kappa_{2}-1}(1-u)^{\kappa_{3}-\kappa_{2}-1} \\
& \times e^{u w} E_{\alpha}\left[-\frac{p}{u(1-u)}\right] d u \\
&\left(\Re\left(\kappa_{3}\right)>\Re\left(\kappa_{2}\right)>0, \alpha \in R^{+}, p \in R_{0}^{+}\right) .
\end{aligned}
$$

Furthermore, different expansions of the Beta, Gauss and confluent hypergeometric functions have been presented and explored by many researchers from time to time (see for details [14], [19], [2], [28], [22], [23], [16] etc).

More recently, Ghayasuddin et al. [10] presented a further extension of the Beta function by involving of the multi-index (2s-parameter) Mittag-Leffler function:

$$
\begin{gathered}
B_{p}^{\left(\lambda_{1}, \cdots, \lambda_{s}, \delta_{1}, \cdots, \delta_{s}\right)}\left(\xi_{1}, \xi_{2}\right)=\int_{0}^{1} u^{\xi_{1}-1}(1-u)^{\xi_{2}-1} \\
\times E_{\left(\frac{1}{\lambda_{i}}\right),\left(\delta_{i}\right)}\left[-\frac{p}{u(1-u)}\right] d u \\
\left(\Re\left(\xi_{1}\right)>0, \Re\left(\xi_{2}\right)>0, \lambda_{i}>0, \delta_{i} \in R, p \geq 0\right) .
\end{gathered}
$$

Here $E_{\left(\frac{1}{\lambda_{i}}\right),\left(\delta_{i}\right)}(u)$ is the multi-index Mittag-Leffler function defined and studied by Kiryakova as follows (see [11], [12], [13]; see also [20], [21], and [33]):

$$
E_{\left(\frac{1}{\lambda_{i}}\right),\left(\delta_{i}\right)}(u)=\sum_{\ell=0}^{\infty} \frac{u^{\ell}}{\Gamma\left(\delta_{1}+\frac{\ell}{\lambda_{1}}\right) \cdots \Gamma\left(\delta_{s}+\frac{\ell}{\lambda_{s}}\right)},
$$

where $s>1$ is an integer, $\lambda_{1}, \cdots, \lambda_{s}>0$ and $\delta_{1}, \cdots, \delta_{s}$ are arbitrary real numbers. Clearly, for $s=2$, if we set $\frac{1}{\lambda_{1}}=\alpha, \frac{1}{\lambda_{2}}=0$, and $\delta_{1}=\delta_{2}=1$ in (20) then we get the extended Beta function $B_{\alpha}^{p}\left(\xi_{1}, \xi_{2}\right)$, given in (14).

Further, setting $\alpha=1,(20)$ reduces to (9), which further for $p=0$ gives the classical Beta function (1).

Ghayasuddin et al. [10] presented the expanded hypergeometric function and expanded confluent hypergeometric function, respectively, by

$$
\begin{gathered}
F_{p}^{\left(\lambda_{1}, \cdots, \lambda_{s}, \delta_{1}, \cdots, \delta_{s}\right)}\left(\kappa_{1}, \kappa_{2} ; \kappa_{3} ; w\right) \\
=\sum_{\ell=0}^{\infty} \frac{\left(\kappa_{1}\right)_{\ell} B_{p}^{\left(\lambda_{1}, \cdots, \lambda_{s}, \delta_{1}, \cdots, \delta_{s}\right)}\left(\kappa_{2}+\ell, \kappa_{3}-\kappa_{2}\right)}{B\left(\kappa_{2}, \kappa_{3}-\kappa_{2}\right)} \frac{w^{\ell}}{\ell !} \\
\quad\left(p \geq 0,|w|<1, \Re\left(\kappa_{3}\right)>\Re\left(\kappa_{2}\right)>0, \lambda_{i}>0, \delta_{i} \in R\right)
\end{gathered}
$$


and

$$
\begin{gathered}
\Phi_{p}^{\left(\lambda_{1}, \cdots, \lambda_{s}, \delta_{1}, \cdots, \delta_{s}\right)}\left(\kappa_{2} ; \kappa_{3} ; w\right) \\
=\sum_{\ell=0}^{\infty} \frac{B_{p}^{\left(\lambda_{1}, \cdots, \lambda_{s}, \delta_{1}, \cdots, \delta_{s}\right)}\left(\kappa_{2}+\ell, \kappa_{3}-\kappa_{2}\right)}{B\left(\kappa_{2}, \kappa_{3}-\kappa_{2}\right)} \frac{w^{\ell}}{\ell !} \\
\quad\left(p \geq 0, \Re\left(\kappa_{3}\right)>\Re\left(\kappa_{2}\right)>0, \lambda_{i}>0, \delta_{i} \in R\right) .
\end{gathered}
$$

Remark 1. It is observed that, for $s=2$, if we set $\frac{1}{\lambda_{1}}=1, \frac{1}{\lambda_{2}}=0$, and $\delta_{1}=\delta_{2}=1$, then (22) and (23) reduce to the known extensions of Gauss and confluent hypergeometric functions defined in [5].

The authors in [10] also established the following integral representations of the expanded Gauss and confluent hypergeometric functions by utilizing (16):

$$
\begin{gathered}
F_{p}^{\left(\lambda_{1}, \cdots, \lambda_{s}, \delta_{1}, \cdots, \delta_{s}\right)}\left(\kappa_{1}, \kappa_{2} ; \kappa_{3} ; w\right)=\frac{1}{B\left(\kappa_{2}, \kappa_{3}-\kappa_{2}\right)} \\
\times \int_{0}^{1} u^{\kappa_{2}-1}(1-u)^{\kappa_{3}-\kappa_{2}-1}(1-u w)^{-\kappa_{1}} E_{\left(\frac{1}{\lambda_{i}}\right),\left(\delta_{i}\right)}\left[-\frac{p}{u(1-u)}\right] d u \\
\left(p \geq 0,|\arg (1-w)|<\pi, \Re\left(\kappa_{3}\right)>\Re\left(\kappa_{2}\right)>0, \lambda_{i}>0, \delta_{i} \in R\right)
\end{gathered}
$$

and

$$
\begin{gathered}
\Phi_{p}^{\left(\lambda_{1}, \cdots, \lambda_{s}, \delta_{1}, \cdots, \delta_{s}\right)}\left(\kappa_{2} ; \kappa_{3} ; u\right)=\frac{1}{B\left(\kappa_{2}, \kappa_{3}-\kappa_{2}\right)} \\
\times \int_{0}^{1} u^{\kappa_{2}-1}(1-u)^{\kappa_{3}-\kappa_{2}-1} e^{u w} E_{\left(\frac{1}{\lambda_{i}}\right),\left(\delta_{i}\right)}\left[-\frac{p}{u(1-u)}\right] d u \\
\left(p \geq 0, \Re\left(\kappa_{3}\right)>\Re\left(\kappa_{2}\right)>0, \lambda_{i}>0, \delta_{i} \in R\right) .
\end{gathered}
$$

The Whittaker functions emerge in relation to the Whittaker differential equation (see [9]). These functions have attained a significant extensions due to their utility in application of mathematics to physical and technical problems. In addition, they are firmly identified with the confluent hypergeometric functions, which assume a significant role in several branches of applied mathematics and theoretical physics for example, fluid mechanics, atomic structure theory and electromagnetic diffraction hypothesis.

The Whittaker function $M_{\eta, \zeta}(w)$ (which is a modified solution of Whittaker equation) with respect to the confluent hypergeometric function of first kind (see [31], [32], see additionally [29]) is defined as

$$
M_{\eta, \zeta}(w)=w^{\zeta+\frac{1}{2}} \quad \exp \left(-\frac{w}{2}\right) \Phi\left(\zeta-\eta+\frac{1}{2} ; 2 \zeta+1 ; w\right)
$$




$$
\left(\eta \in C, 2 \zeta \in C \backslash Z^{-}, \Re(\zeta)>-\frac{1}{2} \quad \text { and } \Re(\zeta \pm \eta)>-\frac{1}{2}\right) .
$$

In 2013, Nagar et al. [18] generalized the Whittaker function of first kind by involving the expanded confluent hypergeometric function $\Phi_{p}$ defined by Chaudhry et al. [5], as follows:

$$
\begin{gathered}
M_{p, \eta, \zeta}(w)=w^{\zeta+\frac{1}{2}} \exp \left(-\frac{w}{2}\right) \quad \Phi_{p}\left(\zeta-\eta+\frac{1}{2} ; 2 \zeta+1 ; w\right) \\
\left(p \geq 0, \eta \in C, 2 \zeta \in C \backslash Z^{-}, \Re(\zeta)>-\frac{1}{2} \text { and } \Re(\zeta \pm \eta)>-\frac{1}{2}\right) .
\end{gathered}
$$

This definition clearly reduces to the simple Whittaker function (26), when we set $p=0$.

\section{A new generalized Whittaker function}

In this section, we consider and investigate a generalization of the expanded Whittaker function related to the expanded confluent hypergeometric function of first kind defined by Ghayasuddin et al. [10]. Some basic properties of this initiated Whittaker function are additionally determined.

Definition 2. The generalized expanded Whittaker function for $\lambda_{i}>$ $0, \delta_{i} \in R$ and $p \geq 0$ is denoted by $M_{\eta, \zeta, p}^{\left(\lambda_{1}, \cdots, \lambda_{s}, \delta_{1}, \cdots, \delta_{s}\right)}(w)$ and defined as

$$
\begin{gathered}
M_{\eta, \zeta, p}^{\left(\lambda_{1}, \cdots, \lambda_{s}, \delta_{1}, \cdots, \delta_{s}\right)}(w) \\
=w^{\zeta+\frac{1}{2}} \exp \left(-\frac{w}{2}\right) \quad \Phi_{p}^{\left(\lambda_{1}, \cdots, \lambda_{s}, \delta_{1}, \cdots, \delta_{s}\right)}\left(\zeta-\eta+\frac{1}{2} ; 2 \zeta+1 ; w\right),
\end{gathered}
$$

where $s>1$ is an integer, $-\pi<\arg (w) \leq \pi, \Re(\zeta)>-\frac{1}{2}, \Re(\zeta \pm \eta)>$ $-\frac{1}{2}, \eta \in C, 2 \zeta \in C \backslash Z^{-}$and $\Phi_{p}^{\left(\lambda_{1}, \cdots, \lambda_{s}, \delta_{1}, \cdots, \delta_{s}\right)}($.$) is the extended confluent$ hypergeometric function of the first kind given in (23).

For $s=2$, by setting $\frac{1}{\lambda_{1}}=1, \frac{1}{\lambda_{2}}=0$, and $\delta_{1}=\delta_{2}=1$ in (28), we get the generalized Whittaker function defined by Nagar et al. [18], which further for $p=0$ gives the usual Whittaker function $M_{\eta, \zeta}(w)$.

Integral representations: By using (20) and (23) in (28), we obtain the following integral representation of our expanded Whittaker function:

$$
M_{\eta, \zeta, p}^{\left(\lambda_{1}, \cdots, \lambda_{s}, \delta_{1}, \cdots, \delta_{s}\right)}(w)=w^{\zeta+\frac{1}{2}} \exp \left(-\frac{w}{2}\right) \frac{1}{B\left(\zeta-\eta+\frac{1}{2}, \zeta+\eta+\frac{1}{2}\right)}
$$




$$
\begin{gathered}
\times \int_{0}^{1} u^{\zeta-\eta-\frac{1}{2}}(1-u)^{\zeta+\eta-\frac{1}{2}} \exp (u w) E_{\left(\frac{1}{\lambda_{i}}\right),\left(\delta_{i}\right)}\left[-\frac{p}{u(1-u)}\right] d u \\
\left(\Re(\zeta)>\Re(\zeta \pm \eta)>-\frac{1}{2} \text { and } \mathrm{p} \geq 0, \lambda_{\mathrm{i}}>0, \delta_{\mathrm{i}} \in \mathrm{R}\right) .
\end{gathered}
$$

If we set the transformation $u=\frac{t-\alpha}{\beta-\alpha}$ in (29), the alternative representation for our newly introduced expanded Whittaker function can be acquired

$$
\begin{gathered}
M_{\eta, \zeta, p}^{\left(\lambda_{1}, \cdots, \lambda_{s}, \delta_{1}, \cdots, \delta_{s}\right)}(w)=(\beta-\alpha)^{-2 \zeta} w^{\zeta+\frac{1}{2}} \exp \left(-\frac{w}{2}\right) \\
\times \frac{1}{B\left(\zeta-\eta+\frac{1}{2}, \zeta+\eta+\frac{1}{2}\right)} \int_{\alpha}^{\beta}(t-\alpha)^{\zeta-\eta-\frac{1}{2}}(\beta-t)^{\zeta+\eta-\frac{1}{2}} \exp \left\{\frac{w(t-\alpha)}{(\beta-\alpha)}\right\} \\
\times E_{\left(\frac{1}{\lambda_{i}}\right),\left(\delta_{i}\right)}\left[-\frac{p(\beta-\alpha)^{2}}{(t-\alpha)(\beta-t)}\right] d t
\end{gathered}
$$

where $\alpha$ and $\beta$ are scalars such that $(\beta-\alpha)>0$.

Taking $\alpha=-1$ and $\beta=1$ in (30), we obtain another integral representation for this expanded Whittaker function:

$$
\begin{gathered}
M_{\eta, \zeta, p}^{\left(\lambda_{1}, \cdots, \lambda_{s}, \delta_{1}, \cdots, \delta_{s}\right)}(w)=2^{-2 \zeta} w^{\zeta+\frac{1}{2}} \frac{1}{B\left(\zeta-\eta+\frac{1}{2}, \zeta+\eta+\frac{1}{2}\right)} \\
\times \int_{-1}^{1}(1+t)^{\zeta-\eta-\frac{1}{2}}(1-t)^{\zeta+\eta-\frac{1}{2}} \exp \left(\frac{w t}{2}\right) E_{\left(\frac{1}{\lambda_{i}}\right),\left(\delta_{i}\right)}\left[-\frac{4 p}{\left(1-t^{2}\right)}\right] d t .
\end{gathered}
$$

Further, on substituting $u=\frac{t}{1+t}$ in (29), we obtain another integral representation for the expanded Whittaker function as follows:

$$
\begin{gathered}
M_{\eta, \zeta, p}^{\left(\lambda_{1}, \cdots, \lambda_{s}, \delta_{1}, \cdots, \delta_{s}\right)}(w)=w^{\zeta+\frac{1}{2}} \exp \left(-\frac{w}{2}\right) \frac{1}{B\left(\zeta-\eta+\frac{1}{2}, \zeta+\eta+\frac{1}{2}\right)} \\
\times \int_{0}^{\infty} t^{\zeta-\eta-\frac{1}{2}}(1+t)^{-(2 \zeta+1)} \exp \left[\frac{w t}{1+t}\right] E_{\left(\frac{1}{\lambda_{i}}\right),\left(\delta_{i}\right)}\left[-\frac{p(1+w)^{2}}{t}\right] d t .
\end{gathered}
$$

For $s=2, \frac{1}{\lambda_{1}}=1, \frac{1}{\lambda_{2}}=0$, and $\delta_{1}=\delta_{2}=1$ in (29)-(32), we obtain the integral representations of the expanded Whittaker function defined by Nagar et al. [18], which further for $p=0$ yields the integral representations for the classical Whittaker function. 


\section{Transformation formula of $M_{\eta, \zeta, p}^{\left(\lambda_{1}, \cdots, \lambda_{s}, \delta_{1}, \cdots, \delta_{s}\right)}(w)$}

Theorem 3. For $p \geq 0$, the following transformation formula holds true:

$$
M_{\eta, \zeta, p}^{\left(\lambda_{1}, \cdots, \lambda_{s}, \delta_{1}, \cdots, \delta_{s}\right)}(-w)=(-1)^{\zeta+\frac{1}{2}} M_{-\eta, \zeta, p}^{\left(\lambda_{1}, \cdots, \lambda_{s}, \delta_{1}, \cdots, \delta_{s}\right)}(w) .
$$

Proof. By utilizing the definition of our expanded Whittaker function $M_{\eta, \zeta, p}^{\left(\lambda_{1}, \cdots, \lambda_{s}, \delta_{1}, \cdots, \delta_{s}\right)}(-w)$, we get

$$
\begin{aligned}
& M_{\eta, \zeta, p}^{\left(\lambda_{1}, \cdots, \lambda_{s}, \delta_{1}, \cdots, \delta_{s}\right)}(-w)=(-w)^{\zeta+\frac{1}{2}} \exp \left(\frac{w}{2}\right) \\
& \times \Phi_{p}^{\left(\lambda_{1}, \cdots, \lambda_{s}, \delta_{1}, \cdots, \delta_{s}\right)}\left(\zeta-\eta+\frac{1}{2} ; 2 \zeta+1 ;-w\right) .
\end{aligned}
$$

If we replace $u$ by $1-u$ in (25), then we obtain

$$
\Phi_{p}^{\left(\lambda_{1}, \cdots, \lambda_{s}, \delta_{1}, \cdots, \delta_{s}\right)}\left(\kappa_{2} ; \kappa_{3} ; w\right)=\exp (w) \Phi_{p}^{\left(\lambda_{1}, \cdots, \lambda_{s}, \delta_{1}, \cdots, \delta_{s}\right)}\left(\kappa_{3}-\kappa_{2} ; \kappa_{3} ;-w\right) .
$$

Applying (35) in the right side of (34) and afterward by using (28), we get the claimed result (33).

\section{Integral transforms of $M_{\eta, \zeta, p}^{\left(\lambda_{1}, \cdots, \lambda_{s}, \delta_{1}, \cdots, \delta_{s}\right)}(w)$}

This section deals with some well-known integral transforms involving the expanded Whittaker function $M_{\eta, \zeta, p}^{\left(\lambda_{1}, \cdots, \lambda_{s}, \delta_{1}, \cdots, \delta_{s}\right)}(w)$, as introduced here.

Theorem 4. For $p \geq 0,(2 \alpha-\beta)>0$ and $\Re(\rho+\zeta)>-\frac{1}{2}$, we have

$$
\begin{aligned}
\int_{0}^{\infty} & w^{\rho-1} \exp (-\alpha w) M_{\eta, \zeta, p}^{\left(\lambda_{1}, \cdots, \lambda_{s}, \delta_{1}, \cdots, \delta_{s}\right)}(\beta w) d w=\frac{\beta^{\zeta+\frac{1}{2}} \Gamma\left(\rho+\zeta+\frac{1}{2}\right)}{\left(\alpha+\frac{\beta}{2}\right)^{\rho+\zeta+\frac{1}{2}}} \\
& \times F_{p}^{\left(\lambda_{1}, \cdots, \lambda_{s}, \delta_{1}, \cdots, \delta_{s}\right)}\left(\zeta+\rho+\frac{1}{2}, \zeta-\eta+\frac{1}{2} ; 2 \zeta+1 ; \frac{2 \beta}{2 \alpha+\beta}\right)
\end{aligned}
$$

where $\left|\arg \left(1-\frac{2 \beta}{2 \alpha+\beta}\right)\right|<\pi$. 
Proof. We begin with L.H.S. of (36) and utilizing the integral representation of the generalized expanded Whittaker function given in (29), we obtain

$$
\begin{gathered}
\int_{0}^{\infty} w^{\rho-1} \exp (-\alpha w) M_{\eta, \zeta, p}^{\left(\lambda_{1}, \cdots, \lambda_{s}, \delta_{1}, \cdots, \delta_{s}\right)}(\beta w) d w \\
=\frac{1}{B\left(\zeta-\eta+\frac{1}{2}, \zeta+\eta+\frac{1}{2}\right)} \int_{0}^{\infty} w^{\rho-1} \exp (-\alpha w)(\beta w)^{\zeta+\frac{1}{2}} \exp \left(-\frac{\beta w}{2}\right) \\
\times\left[\int_{0}^{1} u^{\zeta-\eta-\frac{1}{2}}(1-u)^{\zeta+\eta-\frac{1}{2}} \exp (\beta w u) E_{\left(\frac{1}{\lambda_{i}}\right),\left(\delta_{i}\right)}\left[-\frac{p}{u(1-u)}\right] d u\right] d w .
\end{gathered}
$$

Now rearranging in reference of integration and integrate regarding $w$ by utilizing the definition of usual Gamma function, we obtain

$$
\begin{gathered}
\int_{0}^{\infty} w^{\rho-1} \exp (-\alpha w) M_{\eta, \zeta}^{\left(\lambda_{1}, \cdots, \lambda_{s}, \delta_{1}, \cdots, \delta_{s}\right)}(\beta w) d w \\
=\frac{\beta^{\zeta+\frac{1}{2}} \Gamma\left(\rho+\zeta+\frac{1}{2}\right)}{\left(\alpha+\frac{\beta}{2}\right)^{\rho+\zeta+\frac{1}{2}} B\left(\zeta-\eta+\frac{1}{2}, \zeta+\eta+\frac{1}{2}\right)} \\
\times\left[\int_{0}^{1} u^{\zeta-\eta-\frac{1}{2}}(1-u)^{\zeta+\eta-\frac{1}{2}}\left(1-\frac{2 \beta u}{2 \alpha+\beta}\right)^{-\left(\rho+\zeta+\frac{1}{2}\right)}\right. \\
\left.\times E_{\left(\frac{1}{\lambda_{i}}\right),\left(\delta_{i}\right)}\left[-\frac{p}{u(1-u)}\right] d u\right] .
\end{gathered}
$$

Finally, applying the integral representation (18) in the right side of (37), we show up at our guaranteed outcome (36).

Corollary 5. On setting $\beta=\rho=1$ in (36), we have the following Laplace transform for the expanded Whittaker function:

$$
\begin{gathered}
\int_{0}^{\infty} \exp (-\alpha w) M_{\eta, \zeta, p}^{\left(\lambda_{1}, \cdots, \lambda_{s}, \delta_{1}, \cdots, \delta_{s}\right)}(w) d w=\frac{2^{\zeta+\frac{3}{2}} \Gamma\left(\zeta+\frac{3}{2}\right)}{(2 \alpha+1)^{\zeta+\frac{3}{2}}} \\
\times F_{p}^{\left(\lambda_{1}, \cdots, \lambda_{s}, \delta_{1}, \cdots, \delta_{s}\right)}\left(\zeta+\frac{3}{2}, \zeta-\eta+\frac{1}{2} ; 2 \zeta+1 ; \frac{2}{2 \alpha+1}\right) .
\end{gathered}
$$

Corollary 6. On setting $\beta=2 \alpha$ in (36), we have the following transform for the expanded Whittaker function:

$$
\int_{0}^{\infty} w^{\rho-1} \exp (-\alpha w) M_{\eta, \zeta, p}^{\left(\lambda_{1}, \cdots, \lambda_{s}, \delta_{1}, \cdots, \delta_{s}\right)}(2 \alpha w) d w=\frac{\Gamma\left(\rho+\zeta+\frac{1}{2}\right)}{(2 \alpha)^{\rho}}
$$




$$
\times F_{p}^{\left(\lambda_{1}, \cdots, \lambda_{s}, \delta_{1}, \cdots, \delta_{s}\right)}\left(\zeta+\rho+\frac{1}{2}, \zeta-\eta+\frac{1}{2} ; 2 \zeta+1 ; 1\right),
$$

where $p \geq 0$ and $\Re\left(\rho+\zeta+\frac{1}{2}\right)>0$.

Theorem 7. For $\Re(\zeta \pm \eta)>-\frac{1}{2}$ and $\Re(\zeta+\mu)>-\frac{5}{2}$, the following Hankel transformation holds true:

$$
\begin{gathered}
\int_{0}^{\infty} w M_{\eta, \zeta, p}^{\left(\lambda_{1}, \cdots, \lambda_{s}, \delta_{1}, \cdots, \delta_{s}\right)}(w) J_{\mu}(a w) d w=\frac{\Gamma\left(\zeta+\mu+\frac{5}{2}\right)}{\left(a^{2}+\frac{1}{4}\right)^{\frac{\zeta}{2}+\frac{5}{4}}} \\
\times \sum_{\ell=0}^{\infty} \frac{B_{p}^{\left(\lambda_{1}, \cdots, \lambda_{s}, \delta_{1}, \cdots, \delta_{s}\right)}\left(\zeta-\eta+\frac{1}{2}+\ell, \zeta+\eta+\frac{1}{2}\right)}{B\left(\zeta-\eta+\frac{1}{2}, \zeta+\eta+\frac{1}{2}\right)} \\
\times \frac{\left(\zeta+\mu+\frac{5}{2}\right) \ell}{\left(a^{2}+\frac{1}{4}\right)^{\frac{\ell}{2}} \ell !} P_{\zeta+\ell+\frac{3}{2}}^{-\mu}\left(\frac{1}{\sqrt{4 a^{2}+1}}\right)
\end{gathered}
$$

where $P_{\zeta+\ell+\frac{3}{2}}^{-\mu}(w)$ is the Legendre function [29].

Proof. By utilizing (28) and (23), expanding $M_{\eta, \zeta, p}^{\left(\lambda_{1}, \cdots, \lambda_{s}, \delta_{1}, \cdots, \delta_{s}\right)}(w)$ in terms of generalized expanded beta function and rearranging in reference to integration and summation (which is ensured under the conditions), we acquire

$$
\begin{gathered}
\int_{0}^{\infty} w M_{\eta, \zeta, p}^{\left(\lambda_{1}, \cdots, \lambda_{s}, \delta_{1}, \cdots, \delta_{s}\right)}(w) J_{\mu}(a w) d w \\
=\sum_{\ell=0}^{\infty} \frac{B_{p}^{\left(\lambda_{1}, \cdots, \lambda_{s}, \delta_{1}, \cdots, \delta_{s}\right)}\left(\zeta-\eta+\frac{1}{2}+\ell, \zeta+\eta+\frac{1}{2}\right)}{B\left(\zeta-\eta+\frac{1}{2}, \zeta+\eta+\frac{1}{2}\right) \ell !} \\
\times \int_{0}^{\infty} w^{\zeta+\ell+\frac{3}{2}} \exp \left(-\frac{w}{2}\right) J_{\mu}(a w) d w .
\end{gathered}
$$

On applying the known result [8]

$$
\int_{0}^{\infty} \exp (-s x) x^{\zeta} J_{\mu}(a x) d x=\Gamma(\zeta+\mu+1) r^{-\zeta-1} P_{\zeta}^{-\mu}\left(\frac{s}{r}\right),
$$

(where $\Re(\zeta+\mu)>-1, r=\sqrt{s^{2}+a^{2}}$ and $P_{\zeta}^{-\mu}(w)$ is the Legendre function [29]), in the above expression and after a little simplification, we obtain our needed result (40). 
Remark 8. Note that, if we put $s=2$ with $\frac{1}{\lambda_{1}}=1, \frac{1}{\lambda_{2}}=0$ and $\delta_{1}=$ $\delta_{2}=1$, in (40) then we obtain the under mentioned Hankel transform for the expanded Whittaker function defined by Nagar et al. [18]:

$$
\begin{gathered}
\int_{0}^{\infty} w M_{\eta, \zeta, p}(w) J_{\mu}(a w) d w=\frac{\Gamma\left(\zeta+\mu+\frac{5}{2}\right)}{\left(a^{2}+\frac{1}{4}\right)^{\frac{\zeta}{2}+\frac{5}{4}}} \\
\times \sum_{\ell=0}^{\infty} \frac{B_{p}\left(\zeta-\eta+\frac{1}{2}+\ell, \zeta+\eta+\frac{1}{2}\right)}{B\left(\zeta-\eta+\frac{1}{2}, \zeta+\eta+\frac{1}{2}\right)} \frac{\left(\zeta+\mu+\frac{5}{2}\right)_{\ell}}{\left(a^{2}+\frac{1}{4}\right)^{\frac{l}{2}} \ell !} P_{\zeta+\ell+\frac{3}{2}}^{-\mu}\left(\frac{1}{\sqrt{4 a^{2}+1}}\right) .
\end{gathered}
$$

\section{Derivative of $M_{\eta, \zeta, p}^{\left(\lambda_{1}, \cdots, \lambda_{s}, \delta_{1}, \cdots, \delta_{s}\right)}(w)$}

Theorem 9. The following differential formula for our generalized expanded Whittaker function holds true:

$$
\begin{gathered}
\frac{d^{r}}{d w^{r}}\left[\exp \left(-\frac{w}{2}\right) w^{-\zeta-\frac{1}{2}} M_{\eta, \zeta, p}^{\left(\lambda_{1}, \cdots, \lambda_{s}, \delta_{1}, \cdots, \delta_{s}\right)}(w)\right]=\frac{\left(\zeta-\eta+\frac{1}{2}\right)_{r}}{(2 \zeta+1)_{r}} \\
\times \exp \left(\frac{w}{2}\right) \frac{w^{-\zeta-\frac{r}{2}-\frac{1}{2}}}{(2 \zeta+1)_{r}} M_{\eta-\frac{r}{2}, \zeta+\frac{r}{2}, p}^{\left(\lambda_{1}, \cdots, \lambda_{s}, \delta_{1}, \cdots, \delta_{s}\right)}(w) .
\end{gathered}
$$

where $r=0,1,2, \cdots$

Proof. Here, first we find the $r^{\text {th }}$ derivative of the expanded confluent hypergeometric function $\Phi_{p}^{\left(\lambda_{1}, \cdots, \lambda_{s}, \delta_{1}, \cdots, \delta_{s}\right)}\left(\kappa_{2} ; \kappa_{3} ; w\right)$, which is given as follows (see $[10])$ :

$$
\begin{gathered}
\frac{d^{r}}{d w^{r}}\left[\Phi_{p}^{\left(\lambda_{1}, \cdots, \lambda_{s}, \delta_{1}, \cdots, \delta_{s}\right)}\left(a_{2} ; a_{3} ; w\right)\right] \\
=\frac{\left(a_{2}\right)_{r}}{\left(a_{3}\right)_{r}} \Phi_{p}^{\left(\lambda_{1}, \cdots, \lambda_{s}, \delta_{1}, \cdots, \delta_{s}\right)}\left(a_{2}+r ; a_{3}+r ; w\right) .
\end{gathered}
$$

Now, by applying the definition of the introduced multi-index Whittaker function on the L.H.S. of (42), we obtain

$$
\frac{d^{r}}{d w^{r}}\left[\exp \left(-\frac{w}{2}\right) w^{-\zeta-\frac{1}{2}} M_{\eta, \zeta, p}^{\left(\lambda_{1}, \cdots, \lambda_{s}, \delta_{1}, \cdots, \delta_{s}\right)}(w)\right]
$$




$$
=\frac{d^{r}}{d w^{r}}\left[\Phi_{p}^{\left(\lambda_{1}, \cdots, \lambda_{s}, \delta_{1}, \cdots, \delta_{s}\right)}\left(\zeta-\eta+\frac{1}{2} ; 2 \zeta+1 ; w\right)\right] .
$$

At last, by applying (43) in the above articulation, and then by utilizing the definition of multi-index Whittaker function given in (28), we easily get our claimed result.

\section{Concluding remarks}

In the present study we have established a multi-index Whittaker function related to the expanded confluent hypergeometric function of the first kind [10]. We have also derived some essential properties of this introduced Whittaker function, namely integral representations, a transformation formula, some integral transforms and a differential formula.

Moreover, we discuss now about alternative integral representations of the proposed multi-index Whittaker function.

Note that the multi-index Mittag-Leffler function $E_{\left(\frac{1}{\lambda_{i}}\right),\left(\delta_{i}\right)}(u)$ has the following relations with the Wright hypergeometric function ${ }_{p} \Psi_{q}(u)$ and the Fox $H$-function $H_{p, q}^{m, n}(u)$ (see for example, Kiryakova [12], [13]):

$$
E_{\left(\frac{1}{\lambda_{i}}\right),\left(\delta_{i}\right)}(u)={ }_{1} \Psi_{m}\left[\begin{array}{c}
(1,1) \\
\left(\delta_{i}, \frac{1}{\lambda_{i}}\right)_{1}^{m}
\end{array}\right]
$$

and

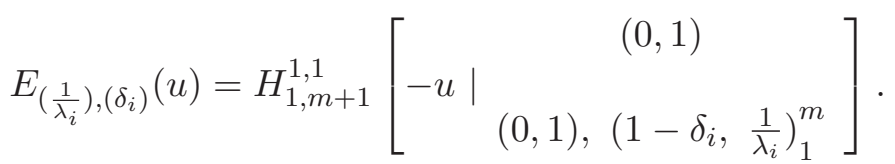

Therefore, the integral representations of our newly expanded Whittaker function given in (29), can be rewritten in terms of the Wright hypergeometric function and Fox $H$-function as follows:

$$
\begin{gathered}
M_{\eta, \zeta, p}^{\left(\lambda_{1}, \cdots, \lambda_{s}, \delta_{1}, \cdots, \delta_{s}\right)}(w)=w^{\zeta+\frac{1}{2}} \exp \left(-\frac{w}{2}\right) \frac{1}{B\left(\zeta-\eta+\frac{1}{2}, \zeta+\eta+\frac{1}{2}\right)} \\
\times \int_{0}^{1} u^{\zeta-\eta-\frac{1}{2}}(1-u)^{\zeta+\eta-\frac{1}{2}} \exp (u w){ }_{1} \Psi_{m}\left[\begin{array}{c}
(1,1) \\
\left.\left(\delta_{i}, \frac{1}{\lambda_{i}}\right)_{1}^{m} \mid \frac{p}{u(1-u)}\right] d u
\end{array}\right.
\end{gathered}
$$


and

$$
\begin{aligned}
& M_{\eta, \zeta, p}^{\left(\lambda_{1}, \cdots, \lambda_{s}, \delta_{1}, \cdots, \delta_{s}\right)}(w)=w^{\zeta+\frac{1}{2}} \exp \left(-\frac{w}{2}\right) \frac{1}{B\left(\zeta-\eta+\frac{1}{2}, \zeta+\eta+\frac{1}{2}\right)} \\
& \times \int_{0}^{1} u^{\zeta-\eta-\frac{1}{2}}(1-u)^{\zeta+\eta-\frac{1}{2}} \exp (u w) \\
& \times H_{1, m+1}^{1,1}\left[-\frac{p}{u(1-u)} \mid(0,1)\right. \\
& \left.(0,1),\left(1-\delta_{i}, \frac{1}{\lambda_{i}}\right)_{1}^{m}\right] d u .
\end{aligned}
$$

\section{References}

[1] M. Ali, M. Ghayasuddin and N.U. Khan, Certain new extension of Whittaker function and its properties, Indian J. Math. 62, No 1 (2020), 81-96.

[2] J. Choi, A.K. Rathie and R.K. Parmar, Extension of extended beta, hypergeometric and confluent hypergeometric functions, Honam Math. J., 36, No 2 (2014), 357-385.

[3] J. Choi, M. Ghayasuddin and N.U. Khan, Generalized extended Whittaker function and its properties, Appl. Math. Sci., 9, No 31 (2015), 6529-6541.

[4] M.A. Chaudhry, A. Qadir, M. Rafique and S.M. Zubair, Extension of Euler's beta function, J. Comput. Appl. Math., 78, No 1 (1997), 19-32.

[5] M.A. Chaudhry, A. Qadir, H.M. Srivastava and R.B. Paris, Extended hypergeometric and confluent hypergeometric functions, Appl. Math. Comput., 159, No 2 (2004), 589-602.

[6] S.A. Dar and R.B. Paris, On the properties of the $(p, \nu)$-extension of the Whittaker function $M_{k, \mu}(z), 2018$; arXiv:1801.09921.

[7] S.A. Dar and M. Shadab, $(p, q)$-extension of the Whittaker function and its certain properties, Commun. Korean Math. Soc., 33, No 2 (2018), 619-630.

[8] A. Erdélyi, W. Magnus, F. Oberhettinger and F.G. Tricomi, Tables of Integral Transforms, Vol. 1, MacGraw-Hill, New York (1954).

[9] A. Erdélyi, W. Magnus, F. Oberhettinger and F.G. Tricomi, Higher Transcendental Functions, Vol. 1, Bateman Manuscript Project, MacGraw-Hill, New York (1953). 
[10] M. Ghayasuddin, N.U. Khan and M. Ali, A study of extended beta, Gauss and confluent hypergeometric function, Intern. J. Appl. Math. 33, No 1 (2020), 1-13; DOI: 10.12732/ijam.v33i1.1.

[11] V.S. Kiryakova, Multiple (multiindex) Mittag-Leffler functions and relations to generalized fractional calculus, J. Comput. Appl. Math. 118 (2000), 241-259.

[12] V. Kiryakova, The multi-index Mittag-Leffler functions as an important class of special functions of fractional calculus, Comput. Math. with Appl. 59 (2010), 1885-1895.

[13] V. Kiryakova, A guide to special functions in fractional calculus, Mathematics, 9, No 1 (2021), Art. 106, 35 pp.; DOI: 10.3390/math9010106.

[14] D.M. Lee, A.K. Rathie, R.K. Parmar and Y.S. Kim, Generalization of extended beta function, hypergeometric and confluent hypergeometric functions, Honam. Math. J., 33, No 2 (2011), 187-206.

[15] Y.L. Luke, The Special Functions and Their Approximations, Vol. 1, New York, Academic Press (1969).

[16] S. Mubeen, G. Rahman, K.S. Nisar, J. Choi and M. Arshad, An extended beta function and its properties, Far East J. Math. Sci., 102, No 7 (2017), $1545-1557$.

[17] G.M. Mittag-Leffler, Sur la représentation analytique d'une fonction monogéne (cinquiéme note), Acta Math., 29, No 1 (1905), 101-181.

[18] D.K. Nagar, R.A.M. Vasquez and A.K. Gupta, Properties of the extended Whittaker function, Progress in Appl. Math., 6, No 2 (2013), 70-80.

[19] E. Özergin, M.A. Özarslan and A. Altin, Extension of gamma, beta and hypergeometric functions, J. Comput. Appl. Math., 235, No 16 (2011), 4601-4610.

[20] J. Paneva-Konovska, A survey on Bessel type functions as multi-index Mittag-Leffler functions: Differential and integral relations, Intern. J. Appl. Math., 32, No 3 (2019), 357-380; DOI: 10.12732/ijam.v32i3.1.

[21] J. Paneva-Konovska, V. Kiryakova, On the multi-index Mittag-Leffler functions and their Mellin transforms, Intern. J. Appl. Math.. 33, No 4 (2020), 549-571; DOI: 10.12732/ijam.v33i4.1. 
[22] P.I. Pucheta, A new extended beta function, Intern. J. Math. Appl., 5, No 3 (2017), 255-260.

[23] R.K. Parmar, A new generalization of gamma, beta, hypergeometric and confluent hypergeometric function, Le Matematiche, LXVIII (2013), 3352 .

[24] R.K. Parmar, P. Chopra and R.B. Paris, On an extension of extended beta and hypergeometric functions, J. Classical Anal., 11, No 2 (2017), 91-106.

[25] E.D. Rainville, Special Functions, The Macmillan Company, New York (1960).

[26] G. Rahman, S. Mubeen, K.S. Nisar and J. Choi, $(p, q)$-Whittaker function and associated properties and formulas, 2017; arXiv:1710.07196.

[27] G. Rahman, K.S. Nisar and J. Choi, An extension of Whittaker function, 2018; arXiv:1801.08032v1.

[28] M. Shadab, S. Jabee and J. Choi, An extension of beta function and its application, Far East J. Math. Sci., 103, No 1 (2018), 235-251.

[29] H.M. Srivastava and H.L. Manocha, A Treatise on Generating Functions, Ellis Horwood Limited, Chichester (1984).

[30] H.M. Srivastava and J. Choi, Zeta and q-zeta Functions and Associated Series and Integral, Elsevier Sci. Publ., Amsterdam, London and New York (2012).

[31] E.T. Whittaker, An expression of certain known functions as generalized hypergeometric functions, Bull. Amer. Math. Soc., 10, No 3 (1903), 125134 .

[32] E.T. Whittaker and G.N. Watson, A Course of Modern Analysis, Reprint of the 4th Ed., Cambridge Math. Library, Cambridge University Press, Cambridge (1990).

[33] S. Yakubovich and Yu. Luchko, The Hypergeometric Approach to Integral Transform and Convolutions, Kluwer Acad. Publ., Dordrecht-Boston, London (1994). 
